\title{
Development of axial asymmetry in the neutron-rich nucleus ${ }^{110} \mathrm{Mo}$
}

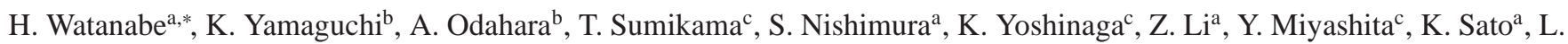

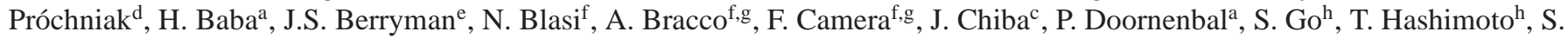
Hayakawa $^{\mathrm{h}}$, C. Hinke ${ }^{\mathrm{i}}$, N. Hinohara ${ }^{\mathrm{a}}$, E. Ideguchi ${ }^{\mathrm{h}}$, T. Isobe ${ }^{\mathrm{a}}$, Y. Ito ${ }^{\mathrm{b}}$, D.G. Jenkins ${ }^{\mathrm{j}}$, Y. Kawada ${ }^{\mathrm{k}}$, N. Kobayashi ${ }^{\mathrm{k}}$, Y. Kondo ${ }^{\mathrm{k}}$, R. Krücken $^{\mathrm{i}}$, S. Kubono ${ }^{\mathrm{h}}$, G. Lorusso ${ }^{1, \mathrm{a}}$, T. Nakano ${ }^{\mathrm{c}}$, T. Nakatsukasa ${ }^{\mathrm{a}}$, M. Kurata-Nishimura ${ }^{\mathrm{a}}$, H.J. Ong ${ }^{\mathrm{m}}$, S. Ota ${ }^{\mathrm{h}}$, Zs. Podolyák ${ }^{\mathrm{n}}$, H. Sakurai $^{\mathrm{a}}$, H. Scheit ${ }^{\mathrm{a}}$, K. Steiger ${ }^{\mathrm{i}}$, D. Steppenbeck ${ }^{\mathrm{a}}$, K. Sugimoto ${ }^{\mathrm{c}}$, K. Tajiri ${ }^{\mathrm{b}}$, S. Takano ${ }^{\mathrm{c}}$, A. Takashima ${ }^{\mathrm{b}}$, T. Teranishi ${ }^{\mathrm{o}}$, Y. Wakabayashi $^{\mathrm{p}}$, P.M. Walker ${ }^{\mathrm{n}}$, O. Wieland ${ }^{\mathrm{f}}$, H. Yamaguchi ${ }^{\mathrm{h}}$

\author{
${ }^{a}$ RIKEN Nishina Center, 2-1 Hirosawa, Wako, Saitama 351-0198, Japan \\ ${ }^{b}$ Department of Physics, Osaka University, Machikaneyama-machi 1-1, Osaka 560-0043 Toyonaka, Japan \\ ${ }^{c}$ Department of Physics, Faculty of Science and Technology, Tokyo University of Science, 2641 Yamazaki, Noda, Chiba, Japan \\ ${ }^{d}$ Institute of Physics, Maria Curie-Skłodowska University, pl. M. Curie-Sktodowskiej 1, 20-031 Lublin, Poland \\ ${ }^{e}$ Nuclear Science Division, Lawrence Berkeley National Laboratory, Berkeley, CA 94720, USA \\ ${ }^{I}$ INFN, Sezione di Milano, via Celoria 16, I-20133 Milano, Italy \\ ${ }^{g}$ Dipartimento di Fisica, Universitá di Milano, via Celoria 16, I-20133 Milano, Italy \\ ${ }^{h}$ Center for Nuclear Study, University of Tokyo, Hirosawa 2-1, Wako, Saitama 351-0198, Japan \\ ${ }^{i}$ Physik Department, Technische Universität München, D-85748 Garching, Germany \\ ${ }^{j}$ Department of Physics, University of York, Heslington, York YO10 5DD, United Kingdom \\ ${ }^{k}$ Department of Physics, Tokyo Institute of Technology, 2-12-1 O-Okayama, Meguro, Tokyo 152-8551, Japan \\ ${ }^{l}$ National Superconducting Cyclotron Laboratory, Michigan State University, East Lansing, MI 48824, USA \\ ${ }^{m}$ Research Center for Nuclear Physics, Osaka University, Ibaraki, Osaka 567-0047, Japan \\ ${ }^{n}$ Department of Physics, University of Surrey, Guildford GU2 7XH, United Kingdom \\ ${ }^{o}$ Department of Physics, Kyushu University, Fukuoka 812-8581, Japan \\ ${ }^{p}$ Japan Atomic Energy Agency, Tokai, Ibaraki 319-1195, Japan
}

\begin{abstract}
The neutron-rich nucleus ${ }^{110} \mathrm{Mo}$ has been investigated by means of $\gamma$-ray spectroscopy following the $\beta$-decay of ${ }^{110} \mathrm{Nb}$, produced using in-flight fission of a ${ }^{238} \mathrm{U}$ beam at $345 \mathrm{MeV} /$ nucleon at the RIBF facility. In addition to the ground-band members reported previously, spectroscopic information on the low-lying levels of the quasi- $\gamma$ band built on the second $2^{+}$state at $494 \mathrm{keV}$ has been obtained for the first time. The experimental finding of the second $2^{+}$state being lower than the yrast $4^{+}$level suggests that axiallyasymmetric $\gamma$ softness is substantially enhanced in this nucleus. The experimental results are compared with model calculations based on the general Bohr Hamiltonian method. The systematics of the low-lying levels in even-even $A \approx 110$ nuclei is discussed in comparison with that in the neutron-rich $A \approx 190$ region, by introducing the quantity $E_{S} / E\left(2_{1}^{+}\right), E_{S}=E\left(2_{2}^{+}\right)-E\left(4_{1}^{+}\right)$, as a global signature of the structural evolution involving axial asymmetry.
\end{abstract}

Keywords: ${ }^{110}$ Mo, Axial asymmetry, Nuclear-shape transitions

\section{Introduction}

One of the long-standing unsolved issues in nuclear structure studies is the rare occurrence of well-deformed oblate (disclike) shapes in nuclear ground states, in contrast to the large abundance of prolate (cigar-like) deformations [1]. A simple explanation for this empirical fact can be suggested in Ref. [2] based on the properties of quantized orbits in deformed potentials. In actual nuclei, however, it is expected that a subtle interplay between the single-particle and collective degrees of freedom plays a significant role in the shape polarization. Under a certain condition that multiple energy minima coexist at prolate and oblate deformation in the potential energy surface [3], the two different shapes can compete, and presumably

\footnotetext{
*Corresponding author

Email address: hiroshi@ribf .riken.jp (H. Watanabe)
}

interact, leading to the nuclear shape being soft with respect to the $\gamma$ degree of freedom, where $\gamma$ represents a deviation from axial symmetry of the ellipsoidal shape; $\gamma=0^{\circ}$ and $60^{\circ}$ for axially-symmetric, prolate and oblate shapes, respectively, and $30^{\circ}$ for a maximally asymmetric nucleus that has three different radii in Cartesian coordinates. The absence of well-deformed oblate ground states in nature may be associated partly with such a transitional character of axially-asymmetric $\gamma$-soft nuclei. Hence, it is certainly necessary to explore deformed nuclei at the critical point of the prolate-to-oblate transition, if a proper understanding of the mechanisms underlying the spontaneous symmetry breaking (Jahn-Teller effect) in nuclear-shape deformation is to be reached.

In the present work, we have investigated neutron-rich $Z \approx$ $40, A \approx 110$ nuclei, in which the phase transitions from prolate, via $\gamma$-soft, to oblate shapes are predicted to occur with increasing number of neutrons [4]. A similar type of the shape evolu- 
tion is suggested for Hf, $\mathrm{W}$, and Os isotopes with $A \approx 190$ [5]. These regions are the ones where the Fermi surfaces for protons and neutrons concurrently lie at the upper halves of the respective major shells, and the rotation alignment of both types of nucleons in the high- $j$ orbits is expected to enhance the oblate stability [6,7]. The recent observation of a possible oblateshape isomer in ${ }^{109} \mathrm{Nb}_{68}$ [8] motivates one to probe the $N=68$ isotone ${ }^{110} \mathrm{Mo}_{68}$, with the particular aim of studying its $\gamma$-soft nature as a fingerprint for the prolate-oblate shape transition.

\section{Experimental procedures}

Experiments were carried out at the RIBF facility [9], cooperated by RIKEN Nishina Center and CNS, University of Tokyo. Neutron-rich $A \approx 110$ nuclei were produced via inflight fission of ${ }^{238} \mathrm{U}^{86+}$ projectiles at $345 \mathrm{MeV} /$ nucleon, incident on a beryllium target with a thickness of $3 \mathrm{~mm}$. The average beam intensity was approximately $0.3 \mathrm{pnA}$ during the experiment. The nuclei of interest were separated and transported through the BigRIPS spectrometer $[10,11]$, operated with a 6 mm-thick wedge-shaped aluminum degrader at the first dispersive focal plane for purification of the secondary beams. An additional degrader placed at the second dispersive focus served as a charge stripper to remove fragments that were not fully stripped. The identification of nuclei by their atomic number and the mass-to-charge ratio was achieved on the basis of the $\Delta E$-TOF- $B \rho$ method, where $\Delta E$, TOF, and $B \rho$ denote energy loss, time of flight, and magnetic rigidity, respectively.

A total of $5.2 \times 10^{4}{ }^{110} \mathrm{Nb}$ ions were implanted into an active stopper consisting of nine double-sided silicon-strip detectors (DSSSD) stacked compactly. Each DSSSD has a thickness of $1 \mathrm{~mm}$ with a $50 \mathrm{~mm} \times 50 \mathrm{~mm}$ active area segmented into sixteen strips on both sides in the vertical and horizontal dimensions. The DSSSDs also served as detectors for electrons following $\beta$-decay and internal-conversion processes. The implantation of an identified particle was associated with the subsequent electron events that were detected in the same DSSSD pixel. Gamma rays were detected by four Compton-suppressed Clover-type Ge detectors arranged around the DSSSD telescope in a close geometry. Further details of a particle-identification spectrum and data-analysis techniques are given in Ref. [8].

\section{Results}

Figure 1 exhibits the level scheme of ${ }^{110} \mathrm{Mo}$, established by means of $\beta$-delayed $\gamma$-ray spectroscopy following the decay of ${ }^{110} \mathrm{Nb}$. Prior to the present work, the ground-state band in ${ }^{110} \mathrm{Mo}$ has been known up to the $10^{+}$state by measuring the prompt $\gamma$ rays from the spontaneous fission of ${ }^{248} \mathrm{Cm}$ [12]. In addition to the 214-, 386-, and 532-keV $\gamma$ rays that belong to the ground-state band, seven new transitions have been unambiguously observed in a singles $\gamma$-ray spectrum measured in coincidence with $\beta$ rays subsequent to implantation of ${ }^{110} \mathrm{Nb}$, as shown in Fig. 2. The main contaminants were from ${ }^{110} \mathrm{Tc}$ [13] and ${ }^{109} \mathrm{Mo}$ [14] succeeding the $\beta$ decay of ${ }^{110} \mathrm{Mo}$ and $\beta$-delayedneutron emission from ${ }^{110} \mathrm{Nb}$, respectively. The efficiency- corrected intensities of $\gamma$-ray transitions and the assigned spins and parities are summarized in Table 1.

A least-squares fit of the summed gated time spectra for the $\gamma$ rays at energies of $281,421,463,487$, and $494 \mathrm{keV}$ yields a half-life of 75(9) ms, which is in agreement with the value of 81(6) ms deduced from the time distribution of the 214-keV $\gamma$ ray within experimental errors, as illustrated in Figs. 3(a) and 3(b). These values are consistent with $T_{1 / 2}=86(6) \mathrm{ms}$ extracted from an independent analysis of $\beta$-decay half-lives [15].

The second $2^{+}$level $\left(2_{2}^{+}\right)$is proposed at $494 \mathrm{keV}$, decaying by the 281- and 494-keV transitions which directly feed the yrast $2^{+}$and $0^{+}$states, respectively. In deformed even-even nuclei, a quasi- $\gamma$ band is built on the low-lying $2_{2}^{+}$state. In the present analysis, the limited statistics preclude us from confirming the coincidence relationship between the 281- and 214-keV $\gamma$ rays. Nevertheless, the assignment of the $2_{2}^{+}$state at $494 \mathrm{keV}$ is justified, since only the energy sum for the $281-214-\mathrm{keV}$ cascade agrees with the energy of the 494-keV $\gamma$ ray within experimental errors among the newly observed $\gamma$ rays. This observation is consistent with the decay pattern from the $2_{2}^{+}$state to the lowerlying $2_{1}^{+}$and $0_{1}^{+}$levels, as confirmed for even ${ }_{42} \mathrm{Mo}$ and ${ }_{44} \mathrm{Ru}$ isotopes in this neutron-rich region [16].

The 207-keV transition is assigned as feeding the $2_{2}^{+}$state on account of the consistency in energy with the level at $701 \mathrm{keV}$, which is most likely the $3^{+}$member of the quasi- $\gamma$ band decaying also via the parallel deexcitation pathway that consists of the 214- and 487-keV transitions. The $\gamma$ rays at 421 and 463 $\mathrm{keV}$ are proposed to be the $4_{2}^{+} \rightarrow 2_{2}^{+}$and $5_{1}^{+} \rightarrow 3_{1}^{+}$transitions, respectively, based on the systematics of the quasi- $\gamma$-band levels for lighter Mo isotopes [16]. The assignment of $5^{+}$for the $1164-\mathrm{keV}$ state is positively supported by the observation of a possible deexcitation to the $4_{1}^{+}$state via the $564-\mathrm{keV} \gamma$ ray. Furthermore, the measured intensity of the 532-keV $\gamma$ ray depopulating the yrast $6^{+}$state at $1132 \mathrm{keV}$ implies a sizable value of spin $(\approx 5 \hbar)$ for the $\beta$-decaying state in ${ }^{110} \mathrm{Nb}$, consistent with the argument above on the population of the $4_{2}^{+}$and $5_{1}^{+}$levels at 916 and $1164 \mathrm{keV}$, respectively, in the $\beta$ decay of ${ }^{110} \mathrm{Nb}$.

The intensities of the $\beta$ feeding to the observed excited states in ${ }^{110}$ Mo can be derived from the measured $\gamma$-ray intensities, taking into account the delayed neutron-emission probability of the ${ }^{110} \mathrm{Nb}$ decay reported in Ref. [17]. The $\beta$-decay branches to the $6_{1}^{+}, 5_{1}^{+}$, and $4_{2}^{+}$states can be estimated to be $13(5), 15(5)$, and $9(4) \%$, respectively, if these levels are directly populated in the $\beta$ decay of ${ }^{110} \mathrm{Nb}$. For the $\beta$ feedings to these high-spin states, the relatively low $\log f t$ values ranging from 5.3 to $5.5^{1}$, which are derived using the half-life of 86(6) ms [15] and mass values evaluated for ${ }^{110} \mathrm{Nb}$ [18] and ${ }^{110} \mathrm{Mo}$ [19], support this spin-parity assignment.

In Fig. 4, the moments of inertia are plotted as a function of the rotational frequency for the ground-state and quasi- $\gamma$ bands of ${ }^{104,106,108,110}$ Mo. Although the proposed level scheme of ${ }^{110}$ Mo depends mainly on the $\gamma$-ray energy matching, the assignment of the quasi- $\gamma$-band levels is reinforced by the obser-

\footnotetext{
${ }^{1}$ Note that the $\log f t$ values obtained in the present work correspond to lower limits on account of possible unobserved $\beta$ feedings to higher-lying levels in ${ }^{110} \mathrm{Mo}$.
} 
vation of the moment of inertia close to that of the ground-state band at low rotational frequencies ( $\hbar \omega \approx 0.2 \mathrm{MeV}$ ), as demonstrated for the lighter Mo isotopes. Similar phenomena have been also reported for ${ }^{110,112} \mathrm{Ru}$ in Ref. [20].

\section{Discussion}

Figure 5(a) exhibits the systematics of the low-lying levels in even ${ }_{42}$ Mo isotopes with neutron numbers ranging from 62 to 68 , including the new result obtained for ${ }^{110} \mathrm{Mo}_{68}$ in the present work. It can be seen that the excitation energies of the $2_{1}^{+}$and $4_{1}^{+}$states reach a minimum at $N=64$; an examination of the $E\left(4_{1}^{+}\right) / E\left(2_{1}^{+}\right)$ratio suggests that the maximum quadrupole deformation of the ground state occurs for ${ }^{106} \mathrm{Mo}_{64}$ [12]. Meanwhile, the $2_{2}^{+}$level falls down in energy as the neutron number increases toward $N=68$. Indeed, the proposed $2_{2}^{+}$state at 494 $\mathrm{keV}$ in ${ }^{110} \mathrm{Mo}$ is lowest in energy of the $2_{2}^{+}$levels identified to date for the even-even nuclei in this neutron-rich region. As mentioned in Sect. 3, the $2_{2}^{+}$states in deformed nuclei are associated predominantly with the bandhead of the quasi- $\gamma$ band. Therefore, the presence of the rather low-lying $2_{2}^{+}$state indicates that the degree of axial asymmetry increases, irrespective of its nature being either dynamic or static.

The experimental excitation energies of the $2_{1}^{+}, 4_{1}^{+}$, and $2_{2}^{+}$ states in even Mo isotopes are reproduced well in a microscopic theory based on the general Bohr Hamiltonian approach [22], in which the potential energy and inertial functions (mass parameters $)^{2}$ are calculated using the constrained Hartree-FockBogoliubov (CHFB) method with the Skyrme effective interaction. For ${ }^{110} \mathrm{Mo}$, the $2_{2}^{+}$state is predicted to lie at about $500 \mathrm{keV}$ (see Fig. 6 in Ref. [22]), being in good agreement with the energy assigned in the present work. The level energies of the ground-state and quasi- $\gamma$ bands calculated using the SIII and SLy4 versions of the Skyrme interaction are shown in Figs. 6(A) and 6(B), respectively.

We also performed a similar calculation with the pairingplus-quadrupole $(\mathrm{P}+\mathrm{Q})$ model including a quadrupole pairing [23]. The parameters in the $\mathrm{P}+\mathrm{Q}$ Hamiltonian are fixed so as to reproduce the Skyrme-HFB calculation with the SLy4 interaction. In this model, the inertial functions are calculated with the local quasiparticle random-phase approximation (LQRPA), in which the contributions from the time-odd mean fields are taken into account, thus the correction factor 1.3 is not necessary [23]. The result of this calculation is shown in Fig. 6(C).

Figure 6 provides the comparison of the experimental levels with the predictions of the two theoretical frameworks. The agreement between the observed and calculated level energies is very satisfactory for both the ground-state and quasi- $\gamma$ bands. In particular, it is noteworthy that the observation for the $2_{2}^{+}$ state being lower than the $4_{1}^{+}$level is reproduced in all of the calculations. The quantity $R_{b}=B\left(E 2 ; 2_{2}^{+} \rightarrow 2_{1}^{+}\right) / B\left(E 2 ; 2_{2}^{+} \rightarrow 0_{1}^{+}\right)$ can be extracted from the measured intensity ratio for a pair of $\gamma$ rays deexciting the $2_{2}^{+}$state on the assumption that the $2_{2}^{+} \rightarrow 2_{1}^{+}$

\footnotetext{
${ }^{2}$ The inertial functions obtained with the Inglis-Belyaev (IB) formula are corrected with a factor of 1.3 [22].
}

transition has a pure $E 2$ multipolarity. In the current work, an experimental value of $R_{b}=10 \pm 3$ has been obtained; this is in reasonable agreement with calculated values, $R_{b}=33.9$ for the CHFB+IB with SLy4 and $R_{b}=9.4$ for the CHFB+LQRPA with $\mathrm{P}+\mathrm{Q}$.

In Fig. 7, the potential energy surface calculations for ${ }^{110} \mathrm{Mo}$ exhibit local minima at the prolate $\left(\gamma \approx 0^{\circ}\right)$ and oblate $\left(\gamma \approx 60^{\circ}\right)$ sides using the SIII and SLy4 versions of the Skyrme interaction, respectively. As mentioned in Ref. [22], however, the exact location of the energy minimum is not important in characterizing the collective level properties of the heavier Mo isotopes, because an overall profile in the potential energy surface spreads over the $\gamma$ degree of freedom. Consequently, the level structure of ${ }^{110} \mathrm{Mo}$ is ascribed to its $\gamma$-soft nature rather than the rigid deformation of any kind.

The systematic behavior of the low-lying level energies in the Mo isotopes compares well with that in neutron-rich ${ }_{74} \mathrm{~W}$ nuclei [21], as is evident from Figs. 5(a) and 5(b); the $2_{2}^{+}$states are lower than the $4_{1}^{+}$levels at $N=68$ and 116 in the Mo and W isotopes, respectively. Such a crossing of the $4_{1}^{+}$and $2_{2}^{+}$states has also been observed for neutron-rich $A \approx 110{ }_{44} \mathrm{Ru}$ nuclei and $A \approx 190{ }_{76}$ Os isotopic chains, in both of which this energy systematics is followed by an increase in the $2_{2}^{+}$energy at neutron numbers higher than 68 and 116 [24, 25].

The comparison of the structural evolution in the $A \approx 110$ and 190 nuclei can be highlighted by introducing the quantity $E_{S} / E\left(2_{1}^{+}\right)$, where $E_{S}$ denotes the energy difference between the $2_{2}^{+}$and $4_{1}^{+}$states. The results are plotted in Figs. 5(c) and 5(d) against the neutron number for selected isotopes in each region. For ${ }_{46} \mathrm{Pd}$ and ${ }_{78} \mathrm{Pt}$ isotopes, which are representative of $\gamma$-soft nuclei $[26,27]$, the deduced values of $E_{S} / E\left(2_{1}^{+}\right)$ are within a narrow range below zero. In the $\gamma$-independent limit of the Wilets-Jean model [28], the quantity $E_{S}$ is zero because the $2_{2}^{+}$and $4_{1}^{+}$states are completely degenerate. On the other hand, the $2_{2}^{+}$state goes under the $4_{1}^{+}$level in the nucleus with a rigid-triaxial shape for $\gamma \geq 25^{\circ}$ in the Davydov and Filippov model [29]; at the extreme of triaxiality $\left(\gamma=30^{\circ}\right)$, $E_{S} / E\left(2_{1}^{+}\right)=-0.67$. Therefore, nuclei with negative values of $E_{S} / E\left(2_{1}^{+}\right)$between these two extremes are most likely characterized by $\gamma$-soft potentials with shallow minima at the average $\gamma$ value close to $30^{\circ}$. This argument on the deformed potential with some $\gamma$ dependence is consistent with the observation of the $3_{1}^{+}$state being depressed relative to the $4_{2}^{+}$level for the $\mathrm{Pd}$ and $\mathrm{Pt}$ isotopes in the regions of interest [16]; these quasi- $\gamma$ band levels would be degenerate in the extreme $\gamma$-independent limit [28].

In Fig. 5(c), a steeper fall of the $E_{S} / E\left(2_{1}^{+}\right)$values is found for the ${ }_{42} \mathrm{Mo}$ isotopes compared to the ${ }_{44} \mathrm{Ru}$ isotopes as the neutron number increases. The lighter isotopes ${ }^{104} \mathrm{Mo}_{62}$ and ${ }^{106} \mathrm{Mo}_{64}$ are known to exhibit rather $\gamma$-vibrational behavior, as demonstrated by the observation of the properties expected for rotational bands built on one- and two- $\gamma$-phonon states [30, 31]. Consequently, the observed rapid decrease in $E_{S} / E\left(2_{1}^{+}\right)$in the Mo isotopic chain reflects the structural change from a nearly axial rotor with the small-amplitude $\gamma$ vibrations to a largeamplitude $\gamma$-soft dynamics. Similar trends can be seen for the ${ }_{74} \mathrm{~W}$ and ${ }_{76} \mathrm{Os}$ isotopes up to $N=116$, but the observed varia- 
tion is more dramatic than that in the $A \approx 110$ nuclei with the same $N$ dispersions, as shown in Fig. 5(d).

One intriguing feature of the empirical $E_{S} / E\left(2_{1}^{+}\right)$plots in Figs. 5(c) and 5(d) is the near coincidence at around -0.5 , observed for both the $N=68$ and 116 isotones. In the case of $N=116$, the heavier isotones ${ }^{192} \mathrm{Os}$ and ${ }^{194} \mathrm{Pt}$ were known to be $\gamma$-soft [32], and the level properties of ${ }^{190} \mathrm{~W}$, such as the anomalous $E\left(4_{1}^{+}\right) / E\left(2_{1}^{+}\right)$ratio approaching the $\gamma$-independent value [21] and isomeric-decay transitions with small $K$ hindrances [33], were considered as evidence for enhanced $\gamma$ softness. For ${ }^{194} \mathrm{Os}_{118}$, however, the sudden change in $E_{S}$ to a positive value was interpreted as an indication of the emergence of an oblate shape [34]. For the $A \approx 110$ nuclei, the second band crossing due to the alignment of a $g_{9 / 2}$ proton pair was observed for the ground-state band in ${ }^{112} \mathrm{Ru}_{68}$, but not in ${ }^{110} \mathrm{Ru}_{66}$ [20], implying a significant change in triaxiality since the crossing frequency for the aligned $\pi g_{9 / 2}$ orbitals is sensitive to the $\gamma$ degree of freedom [4]. These band-crossing phenomena are reproduced well by a recent calculation based on the total-Routhian-surface (TRS) method [35], which indicates that a ground-state transition from triaxial-prolate to triaxialoblate shapes takes place at around $N=68$ in the Ru isotopes as the number of neutrons increases. Thus, we may say that the empirical value of $E_{S} / E\left(2_{2}^{+}\right) \approx-0.5$ is characteristic of the critical-point nuclei in terms of maximum $\gamma$ softness between prolate and oblate shapes. It should be noted that a critical point of a prolate-oblate phase transition in $\gamma$-soft nuclei is discussed in the context of the $\mathrm{O}(6)$ limit of the interacting boson model $[36,37]$. However, to elucidate the relation between the systematic behavior of $E_{S} / E\left(2_{1}^{+}\right)$and the underlying cause for the structural evolutions, further theoretical investigations are necessary.

Finally, it is noteworthy that the quantity $E_{S} / E\left(2_{1}^{+}\right)$serves as a global signature of the phase transitions involving the $\gamma$ degree of freedom. As already mentioned, the nuclei of enhanced $\gamma$ softness take $E_{S} / E\left(2_{1}^{+}\right) \approx-0.5$. Although the quantity $E_{S}$ is likely to be negative for both the $\gamma$-soft and sphericalvibrational nuclei, the former can be distinguished from the latter with the $E_{S} / E\left(2_{1}^{+}\right)$value being, in the latter case, nearly zero since then $E\left(2_{1}^{+}\right)$is rather high. Meanwhile, the value of $E_{S} / E\left(2_{1}^{+}\right)$rises increasingly positive in an axially-symmetric rotor, because the $2_{2}^{+}$state lies at high excitation energy relative to the $2_{1}^{+}$and $4_{1}^{+}$states. The experimental values of $E_{S} / E\left(2_{1}^{+}\right)$are plotted for a broad range of even-even nuclei with $42 \leq Z \leq 58$ and $56 \leq N \leq 78$ in Fig. 8. The transitional features for the sequence of $N=68$ isotones are remarkable; the structure changes from spherical to well-deformed prolate shapes for $Z=50 \rightarrow 58$, while the development of axiallyasymmetric $\gamma$ softness proceeds with decreasing proton number down to 42.

\section{Conclusions}

The level structure of ${ }^{110} \mathrm{Mo}$ has been investigated following the $\beta$-decay of ${ }^{110} \mathrm{Nb}$, populated via in-flight fission of a ${ }^{238} \mathrm{U}$ beam. In addition to the known levels of the ground-state band, several new levels, including a candidate for the $2_{2}^{+}$quasi- $\gamma$ band state, have been identified. This is the most neutron-rich Mo isotope for which spectroscopic information on the lowlying level structure has been obtained. The observed level energies and the $B(E 2)$ ratio for a pair of $\gamma$ rays from the $2_{2}^{+}$ state are reproduced well by the model calculations based on the general Bohr Hamiltonian approach. The potential energy surface calculations indicate that the low-lying level structure of ${ }^{110} \mathrm{Mo}$ is of a $\gamma$-soft nature. The energy systematics of the lowlying levels in $A \approx 110$ Mo isotopes compares well with that in $A \approx 190 \mathrm{~W}$ isotopes. The enhancement of axially-asymmetric $\gamma$ softness in ${ }^{110} \mathrm{Mo}$ is illuminated with the empirical plot of the quantity $E_{S} / E\left(2_{1}^{+}\right), E_{S}=E\left(2_{2}^{+}\right)-E\left(4_{1}^{+}\right)$. In future experiments, it will be of particular interest to investigate the level properties of heavier Mo isotopes, in which more stable oblate shapes are predicted.

\section{Acknowledgements}

We are indebted to the staff members of RIKEN Nishina Center for providing the uranium beams and to the BigRIPS team for tuning the secondary beams. H.W. thanks Prof. I. Hamamoto, Prof. Y. Sun, and Prof. K. Matsuyanagi for valuable discussions. This work was supported by the KAKENHI (Grant Nos. 19340074, 50126124, and 21340073), the RIKEN President's Fund (2005), UK STFC and AWE plc., the DFG Cluster of Excellence Origin and Structure of the Universe and under DFG grant KR 2326/2. The numerical calculation was performed in part on RIKEN Integrated Cluster of Clusters (RICC).

\section{References}

[1] A. Bohr and B. R. Mottelson, Nuclear Structure, vol. II, Benjamin, Reading, MA, 1975.

[2] R. F. Casten, Nuclear Structure from Simple Perspective, Oxford University Press, Oxford, 2000.

[3] J. L. Wood et al., Phys. Rep. 215 (1992) 101.

[4] J. Skalski et al., Nucl. Phys. A 617 (1997) 282.

[5] P. Sarriguren et al., Phys. Rev. C 77 (2008) 064322.

[6] F. R. Xu et al., Phys. Rev. C 65 (2002) 021303.

[7] F. R. Xu et al., Phys. Rev. C 62 (2000) 014301.

[8] H. Watanabe et al., Phys. Lett. B 696 (2011) 186.

[9] Y. Yano, Nucl. Instrum. Methods B 261 (2007) 1009.

[10] T. Kubo, Nucl. Instrum. Methods B 204 (2003) 97.

[11] T. Ohnishi et al., J. Phys. Soc. Jpn 77 (8) (2008) 083201.

[12] W. Urban et al., Eur. Phys. J. A 20 (2004) 381.

[13] J. C. Wang et al., Eur. Phys. J. A 19 (2004) 83.

[14] W. Urban et al., Phys. Rev. C 73 (2006) 037302.

[15] S. Nishimura et al., Phys. Rev. Lett. 106 (2011) 052502.

[16] http://www.nndc.bnl.gov/ensdf/

[17] T. Mehren et al., Phys. Rev. Lett. 77 (1996) 458.

[18] G. Audi et al., Nucl. Phys. A 729 (2003) 337.

[19] U. Hager et al., Phys. Rev. Lett. 96 (2006) 042504

[20] C. Y. Wu et al., Phys. Rev. C 73 (2006) 034312.

[21] N. Alkhomashi et al., Phys. Rev. C 80 (2009) 064308.

[22] L. Próchniak, Int. J. Mod. Phys. E 19 (2010) 705.

[23] N. Hinohara et al., Phys. Rev. C 82 (2010) 064313.

[24] J. A. Shannon et al., Phys. Lett. B 336 (1994) 136.

[25] C. Wheldon et al., Phys. Rev. C 63 (2000) 011304

[26] K.-H. Kim et al., Nucl. Phys. A 604 (1996) 163.

[27] R. F. Casten et al., Nucl. Phys. A 309 (1978) 477. 
[28] L. Wilets et al., Phys. Rev. 102 (1956) 788.

[29] A. S. Davydov et al., Nucl. Phys. 8 (1958) 237 - 249.

[30] A. Guessous et al., Phys. Rev. C 53 (1996) 1191.

[31] A. Guessous et al., Phys. Rev. Lett. 75 (1995) 2280

[32] C. Y. Wu et al., Nucl. Phys. A 607 (1996) 178.

[33] G. J. Lane et al., Phys. Rev. C 82 (2010) 051304.

[34] R. F. Casten et al., Phys. Lett. B 76 (1978) 280.

[35] J. Q. Faisal et al., Phys. Rev. C 82 (2010) 014321.

[36] J. Jolie et al., Phys. Rev. Lett. 87 (2001) 162501.

[37] J. Jolie et al., Phys. Rev. C 68 (2003) 031301.

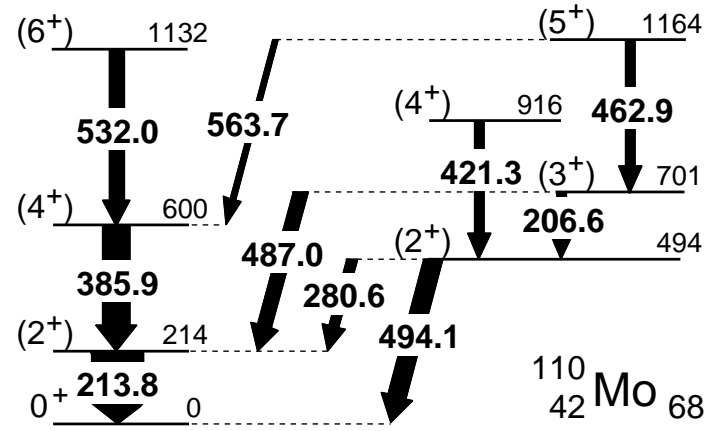

Figure 1: Partial level scheme of ${ }^{110}$ Mo established in the present work. The widths of arrows represent relative intensities of $\gamma$ rays summarized in Table 1 .

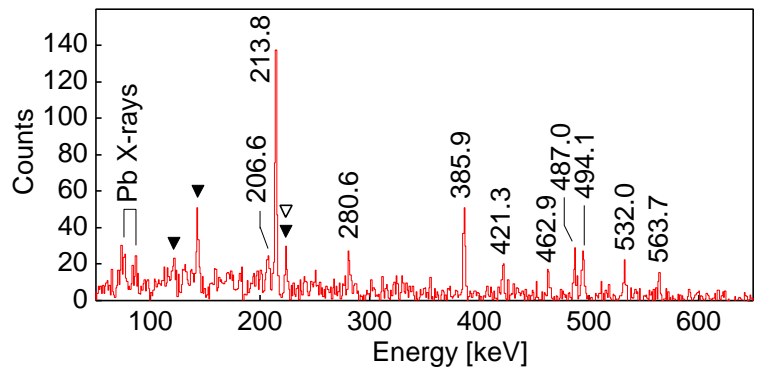

Figure 2: (Color online). $\gamma$-ray energy spectrum measured in coincidence with $\beta$ rays detected within $250 \mathrm{~ms}$ after implantation of ${ }^{110} \mathrm{Nb}$. Contaminants from ${ }^{110} \mathrm{Tc}$ and ${ }^{109} \mathrm{Mo}$ are indicated with filled and open triangles, respectively.

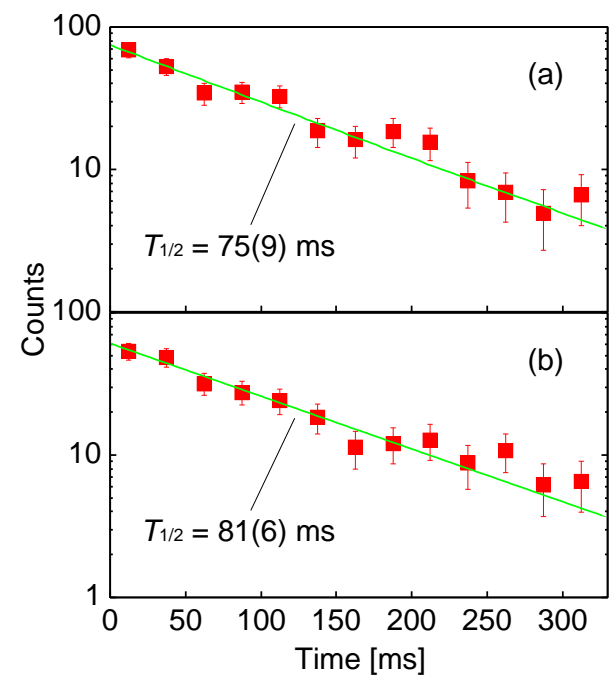

Figure 3: (Color online). Time distributions and associated fits for $\gamma$-ray coincidence events relative to implantation of ${ }^{110} \mathrm{Nb}$ with (a) a sum of gates on the 281-, 421-, 463-, 487-, and 494-keV transitions and (b) a gate on the 214-keV transition in ${ }^{110} \mathrm{Mo}$. 


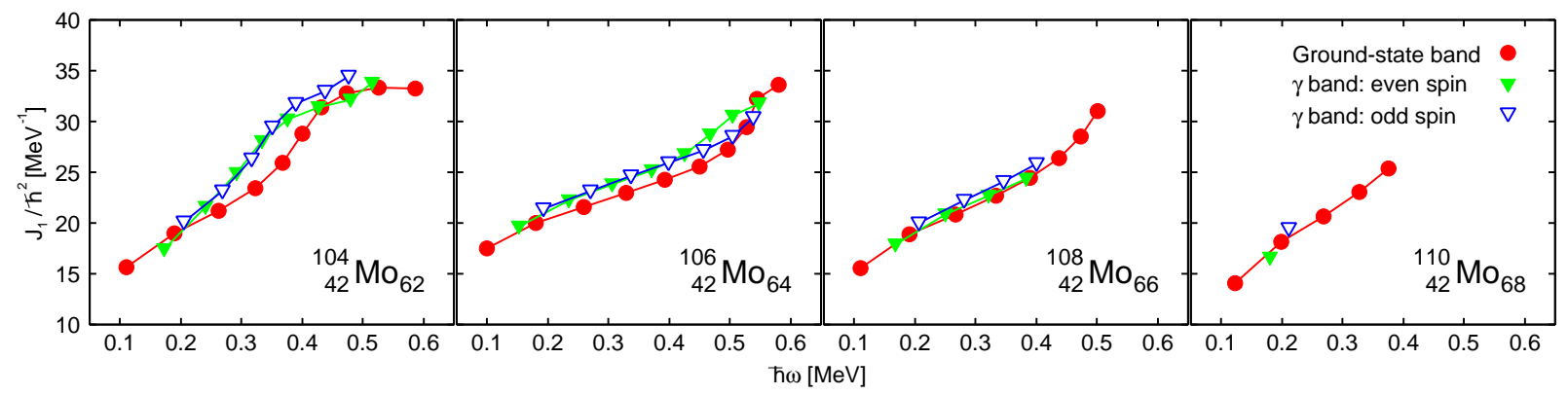

Figure 4: (Color online). Kinematic moment of inertia as a function of the rotational frequency for the ground-state and quasi- $\gamma$ bands in ${ }^{104-110}$ Mo. These data are from Ref. [16] and the present work.

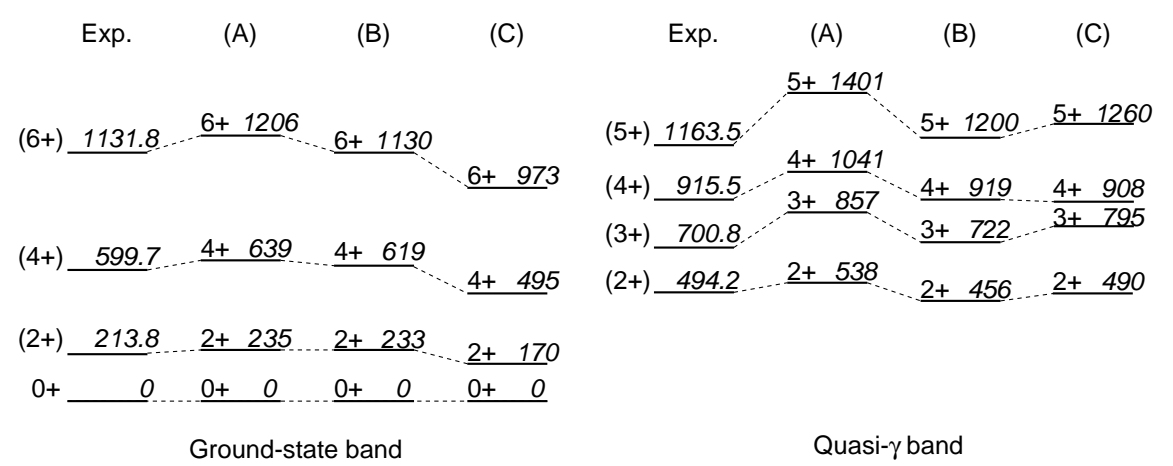

Figure 6: Experimental and calculated level energies (in keV) for the ground-state and quasi- $\gamma$ bands in ${ }^{110}$ Mo. The model calculations are based on the general Bohr Hamiltonian approach with the CHFB+IB method using the SIII (A) and SLy4 (B) versions of the Skyrme interaction, and the CHFB+LQRPA method using the $\mathrm{P}+\mathrm{Q}$ force $(\mathrm{C})$.

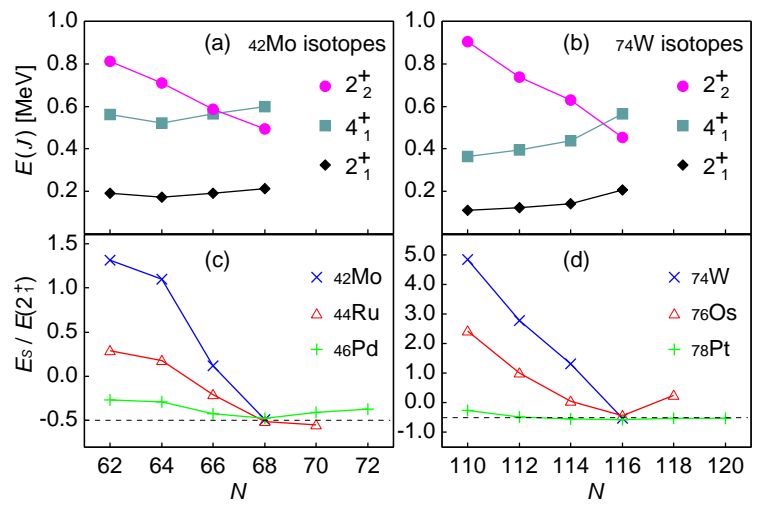

Figure 5: (Color online). Systematics of low-lying levels for even-even nuclei for the (left) $A \approx 110$ region and (right) $A \approx 190$ region as a function of the neutron number. Panels (a) and (b) show the excitation energies of the $2_{1}^{+}, 4_{1}^{+}$, and $2_{2}^{+}$states in ${ }_{42} \mathrm{Mo}$ and ${ }_{74} \mathrm{~W}$ isotopes, respectively. The empirical values of the quantity $E_{S} / E\left(2_{1}^{+}\right)$, where $E_{S}=E\left(2_{2}^{+}\right)-E\left(4_{1}^{+}\right)$, are plotted for (c) ${ }_{42} \mathrm{Mo}$, ${ }_{44} \mathrm{Ru}$, and ${ }_{46} \mathrm{Pd}$ isotopes and (d) ${ }_{74} \mathrm{~W},{ }_{76} \mathrm{Os}$, and ${ }_{78} \mathrm{Pt}$ isotopes, with the dashed lines at -0.5 . These data are from Refs. $[16,21]$ and the present work.
Table 1: Summary of transitions in ${ }^{110}$ Mo observed in the $\beta$-decay of ${ }^{110} \mathrm{Nb}$.

\begin{tabular}{cccc}
\hline \hline $\begin{array}{c}E_{x} \\
(\mathrm{keV})\end{array}$ & $\begin{array}{c}E_{\gamma} \\
(\mathrm{keV})\end{array}$ & $\begin{array}{c}I_{\gamma} \\
\text { (relative) }\end{array}$ & $\begin{array}{c}\text { Assignment } \\
J_{i}^{\pi} \rightarrow J_{f}^{\pi}\end{array}$ \\
\hline $213.8(1)$ & $213.8(1)$ & $100(12)$ & $2_{1}^{+} \rightarrow 0_{1}^{+}$ \\
$494.2(1)$ & $280.6(2)$ & $21(5)$ & $2_{2}^{+} \rightarrow 2_{1}^{+}$ \\
& $494.1(2)$ & $38(7)$ & $2_{2}^{+} \rightarrow 0_{1}^{+}$ \\
$599.7(1)$ & $385.9(1)$ & $56(9)$ & $4_{1}^{+} \rightarrow 2_{1}^{+}$ \\
$700.8(1)$ & $206.6(2)$ & $18(4)$ & $3_{1}^{+} \rightarrow 2_{2}^{+}$ \\
& $487.0(2)$ & $30(6)$ & $3_{1}^{+} \rightarrow 2_{1}^{+}$ \\
$915.5(2)$ & $421.3(2)$ & $21(6)$ & $4_{2}^{+} \rightarrow 2_{2}^{+}$ \\
$1131.8(2)$ & $532.0(2)$ & $29(6)$ & $6_{1}^{+} \rightarrow 4_{1}^{+}$ \\
$1163.5(2)$ & $462.9(3)$ & $22(6)$ & $5_{1}^{+} \rightarrow 3_{1}^{+}$ \\
& $563.7(2)$ & $12(5)$ & $5_{1}^{+} \rightarrow 4_{1}^{+}$ \\
\hline \hline
\end{tabular}



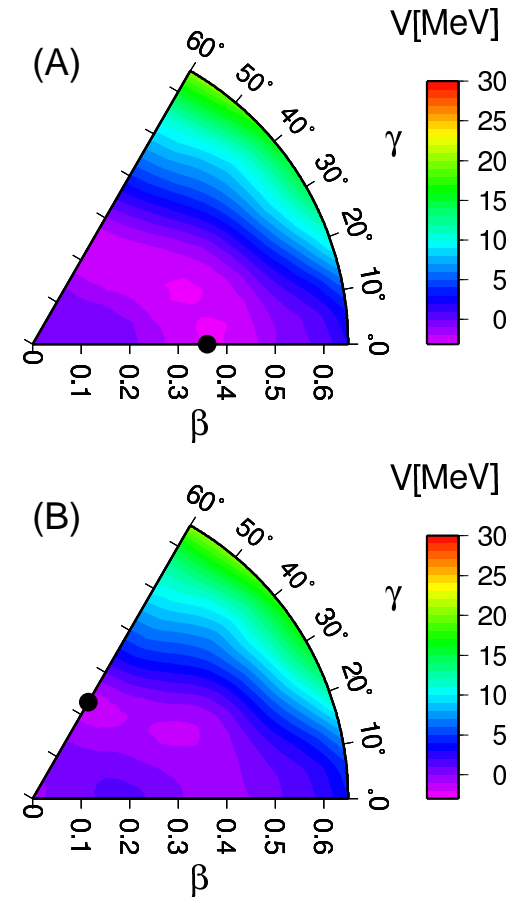

Figure 7: (Color). Potential energy surface calculations for ${ }^{110}$ Mo using the CHFB+IB method with the SIII (A) and SLy4 (B) versions of the Skyrme interaction. The energy minima are indicated with filled circles.

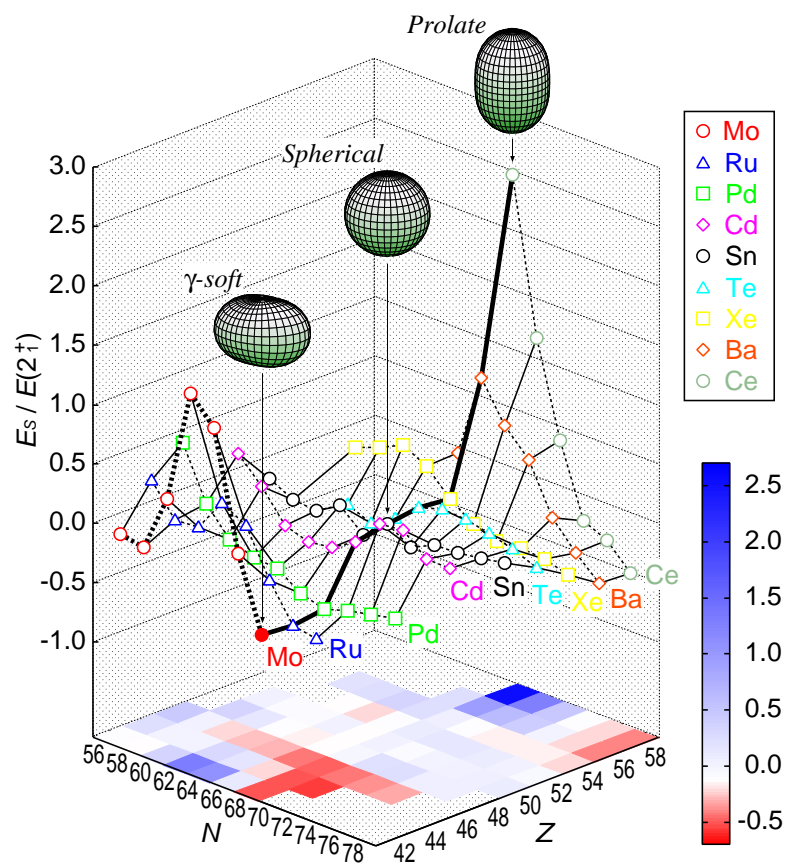

Figure 8: (Color). The landscape of the experimental quantity $E_{S} / E\left(2_{1}^{+}\right)$for even-even $Z=42-58$ nuclei with $N=56-78$. These values are from Ref. [16] (open symbols) and the present work for ${ }^{110} \mathrm{Mo}$ (closed circle). Solid lines link isotones, while dashed lines correspond to isotopic chains; the sequences of $N=68$ and $Z=42$ are thickened for convenience. The $N=68$ isotones that are expected to have axially-symmetric (prolate), spherical, and $\gamma$-soft shapes are indicated by vertical arrows. The data are mapped in the $\mathrm{N}-\mathrm{Z}$ plane at the bottom of the figure. 